\title{
Improving outcomes of hospitalized patients: the Physician Relationships, Improvising, and Sensemaking intervention protocol
}

\author{
Luci K Leykum ${ }^{1,2,3^{*}}$, Holly J Lanham ${ }^{1,2,3}$, Shannon M Provost ${ }^{3}$, Reuben R McDaniel $\mathrm{Jr}^{3}$ and Jacqueline Pugh ${ }^{1,2}$
}

\begin{abstract}
Background: Our goal is to improve the safety and effectiveness of inpatient care. Rather than focus on improving process of care, we focus on the social structure within physician teams. We have developed the Physician Relationships, Improvising, and Sensemaking (PRISm) intervention to improve the way physician teams round, enabling them to better relate, make sense of their patients' conditions, and improvise in uncertain clinical situations. We are currently studying the impact of PRISm on adverse events and complications in hospitalized patients. This manuscript describes the PRISm intervention.

Methods/design: PRISm is a structured communication tool consisting of three components: daily briefings before rounds; use of the Situation, Task, Intent, Concern, and Calibrate (STICC) framework during rounds as part of the discussion of individual patients; and debriefings after rounds. We are implementing the PRISm intervention on eight inpatient medical and surgical physician teams in the South Texas Veterans Health Care System. We are assessing PRISm impact on the way team members relate to each other, round, and discuss patients through pre- and post-implementation observations and surveys. We are also assessing PRISm impact on complications and adverse events. Finally, we are interviewing physicians regarding their experience using the intervention.

Discussion: Our results will allow us to begin to understand the potential impact of interventions designed to improve how providers relate to each other, improvise, and make sense of what is happening as a strategy for improving inpatient care. Our in-depth data collection will enable us to assess how relationships, improvising, and sensemaking influence patient outcomes, potentially through creating shared mental models or enhancing distributed cognition during clinical reasoning. Finally, our results will lay the groundwork for larger implementation studies to improve clinical outcomes through improving how providers, and providers, patients, and caregivers, relate.
\end{abstract}

Keywords: Complexity science, Inpatient care, Physician teams

\section{Background}

Since the Institute of Medicine Report "To Err is Human", increased attention has been paid to improving the care of hospitalized patients [1]. Specific improvement strategies include utilization of guidelines and pathways and the application of quality improvement techniques to improve processes. Despite improvements in focused areas such as prevention of hospital-acquired infections, evidence suggests that systematic improvements in outcomes of

\footnotetext{
* Correspondence: Leykum@uthscsa.edu

'South Texas Veterans Health Care System, Texas, USA

${ }^{2}$ School of Medicine, University of Texas Health Science Center at San Antonio, 7703 Floyd Curl Drive, 373 L, San Antonio, Texas 78229, USA Full list of author information is available at the end of the article
}

hospitalized patients have not been achieved [2]. Rates of errors and hospital-related complications such as falls, decubitus ulcers, and hospital-acquired infections remain high [3-5], and not all patients receive the care known to be appropriate for their illnesses [6]. The costs of these complications are substantial [7].

To date, many attempts to improve inpatient care have used pathway and process-improvement approaches, focusing on impacting the behavior of single individuals or on breaking down processes into component parts. An alternative approach for improving clinical systems is grounded in the framework of complexity science $[8,9]$. Recognizing the complexity of clinical systems provides new insights into the system characteristics to which we 
must pay attention to improve outcomes. First, nonlinearity is a hallmark of complex systems. Inputs and outputs are not necessarily proportional or predictable [10]. The presence of unpredictability introduces the key notion of uncertainty [11-13]. To improve clinical system performance, we must improve providers' ability to perform effectively in the face of uncertainty. This may be particularly true in inpatient environments, where patients are acutely ill, diagnoses are often uncertain, and the possibility of developing complications is significant. In these situations, the uncertainty is compounded: it is inherent in the trajectory of the patient's illness, the limits of our scientific knowledge, and in the system itself $[13,14]$.

The application of complexity science also provides the insight that we must understand the system not only in terms of processes of care but also in terms of interdependencies. While these interdependencies include the processes of care and resources available, they also include the social structure and relationships among providers. The relationship infrastructure is crucial to managing uncertainty because relationships are the foundation for interactions that lead to effective action.

It is through their relationships that people are able to make sense of the uncertain world around them, assimilating information to form conclusions that lead to action. "Sensemaking is a diagnostic process directed at constructing plausible interpretations of ambiguous cues that are sufficient to sustain action" [15]. Making a diagnosis may be part of sensemaking in that it is a process through which providers understand patients' illnesses. However, the scope of sensemaking is larger than making a diagnosis, as it includes taking into account the overall trajectory of a patient's illness over time-particularly recognizing when a change occurs [16]. For example, surgical mortality has been found to be related not to the occurrence of complications but to the ability of the care team to recognize the complication and act effectively [17]. This inability has been called "failure to rescue" [17] and we believe reflects a failure of the team to make sense of a complication.

Improvising is varying what one does based on the context and situation at hand $[18,19]$. Jazz ensembles are frequently used to illustrate improvisation, as each member of the group builds on his or her own talents and experiences as well as those of others, creating an interplay that utilizes the strength of each participant to create a more effective whole [20]. It is important to note that improvising is grounded in knowledge base and skill. In interviews, physicians describe improvising as a key activity in patient care when uncertain or new situations and presentations arise [19].

While physicians may not pay explicit attention to relationships, improvising, and sensemaking, more and more data speaks to their importance. Relationships among surgical team members are associated with their ability to successfully implement new techniques [21]. Primary care clinic staff member relationships are important to clinic function [22], and improving how clinic members in primary care settings speak to each other leads to improved clinic performance $[23,24]$. Literature related to ICU team performance is rooted in characteristics of relationships among team members such as mindfulness [25]. Finally, our own work observing physician teams in inpatient settings demonstrated an association between relationships, sensemaking, and length of stay, unnecessary length of stay, and complication rates [26,27]. In settings from operating rooms [21] to intensive care units [25], and from nursing homes [28] to primary care clinics [20,29], when health care providers are able to make sense of their patients' conditions, care improves.

We seek to improve the outcomes of hospitalized patients through improving physicians' relationships, improvising, and sensemaking. In this manuscript, we describe our protocol for improving physicians' social interactions-the Physician Relationships, Improvising, and Sensemaking (PRISm) intervention.

\section{Methods/design}

\section{General approach}

PRISm is a structured communication intervention grounded in our observations of inpatient teams. [26] It is intended to be integrated into physician rounds. PRISm has three components: briefings before rounds, debriefings after rounds, and a structured tool to be used in discussions of individual patients. We are piloting PRISm on inpatient medicine and surgery teams to assess its impact on team sensemaking behaviors and adverse events. We are observing teams for 1 week to assess team relationships, improvising, and sensemaking. At the end of this week, we are orienting attending physicians to the PRISm intervention and observing the team's implementation of PRISm for an additional week post-intervention.

\section{Briefings}

The purpose of the briefings is to explicitly consider the needs of the group of patients admitted to the team prior to rounding. Our observational work, and writings in the lay press, suggests that rounds are not always conducted based on the needs of patients. The team may not see the sickest patients or those with time-sensitive needs until later in rounds and may not have paced themselves to allow adequate time for these discussions. Additionally, teams may begin rounds only to realize that key information is not available until after they begin discussing a patient. Realizing this prior to rounds could lead them to take actions such as bringing the computer on wheels to rounds or making a phone call 
to obtain crucial information. Thus, briefings are an opportunity for the team to think about their group of patients, assess their overall needs, and determine how best to address them on rounds. Specific areas of discussion (i.e., the "checklist" to guide the discussion) include the following:

- identifying the sickest patients or those with a change in clinical status

- admission/discharge priorities

- assessment of whether team has information required for rounds

\section{Debriefings}

Debriefings are an opportunity for the team to reflect on rounds and the tasks and activities that arose from patient discussions. This reflection is increasingly important in an era when not all team members are present every day and when daytime team members are not the only ones caring for the patient. Because of work hour limitations and outpatient training requirements, team members are increasingly covered by other daytime team members or by "night float" services. Thus, transitions, handoffs, and team members "covering for each other" have become the norm. Explicitly discussing what needs to be accomplished and who is responsible will increase the effectiveness of the team as a cohesive group. Specific areas of discussion (again, the "checklist") for the debriefing include the following:

- recapping the list of most critical tasks, outlining responsibility for each. PRISm will deconstruct the current team hierarchy. Rather than viewing tasks as belonging to "the intern," the debriefing will move the focus to how the team as a whole can get things done, or from individual to group responsibility. For example, it will no longer be acceptable for the intern to say "I didn't get to that yesterday," because the team as a whole has responsibility. This may require higher-level team members to do work normally done by lower-level members. Providers may be more likely to perform activities outside of their expected roles if they better understand the work required. This understanding reflects heedfulness and mindfulness [22].

- developing the list of contingency "if-then" type statements to guide team members' sensemaking as new data becomes available. This is increasingly important for anticipating events that could happen when the team is not present and could serve as a guide for covering providers. To use the analogy of a network, when one node is out, the network must still function. Being explicit about what to anticipate and how to react in certain circumstances will help covering providers to more effectively care for patients.

- need for discussion with others involved in the care of the patient, such as nursing staff, social work, or consultants. Managing these "loose connections" outside of the team is difficult. There may not be set-aside time for different groups to speak. Because of this, communication often occurs through lean media such as notes rather than through rich conversation [10]. One goal of the debriefing is to make the need for verbal communication more explicit.

\section{Framework for individual patient discussions}

We utilized the Situation, Task, Intent, Concern, and Calibrate (STICC) framework as a tool that teams could use as part of their discussions of individual patients [30]. This framework has been used to understand communication failures in inpatient medicine settings [30]. The STICC elements are defined in Table 1 and are discussion points that could be applied to specific patients. STICC is not a replacement for the usual new patient and follow-up presentations that occur on rounds. Instead, its use would augment those discussions, being incorporated into elements of the assessment and plan for individual patients. In the pocket guide, we have suggested specific questions that attendings can use to integrate STICC.

\section{Physician orientation}

To orient attendings to the PRISm intervention, we are utilizing a one-page PRISm information sheet and a pocket guide. The information sheet (Table 2) provides a brief introduction to PRISm and the rationale for its use. It frames PRISm as a structured communication tool based on effective team behaviors, explains how it can be integrated into current rounding practices, and suggests specific ways for implementing the structured discussions. It also specifies time expectations. Our goal is to limit

\section{Table 1 STICC elements and definitions}

\begin{tabular}{|c|c|}
\hline Element & Definition \\
\hline \multirow[t]{2}{*}{ Situation } & Discussion of "here is what we are dealing with". \\
\hline & Working diagnosis \\
\hline \multirow[t]{2}{*}{ Task } & Assessment of "what we are going to do". \\
\hline & Specific next steps should be explicitly discussed. \\
\hline Intent & $\begin{array}{l}\text { Explicit, concrete discussion of why the team is } \\
\text { embarking on a specific diagnostic or therapeutic plan. }\end{array}$ \\
\hline \multirow[t]{2}{*}{ Concern } & $\begin{array}{l}\text { Discussion of "what we need to keep our eye on" } \\
\text { or "what we need to look out for". }\end{array}$ \\
\hline & $\begin{array}{l}\text { Should be specific to the patient, not only general } \\
\text { to the disease. }\end{array}$ \\
\hline Calibrate & $\begin{array}{l}\text { "Talk to me". Discussion regarding what the team might } \\
\text { be missing, what is unclear or not yet understood. }\end{array}$ \\
\hline & If-then contingency statements. \\
\hline
\end{tabular}


Table 2 PRISm background information and implementation sheet

\begin{tabular}{|c|c|}
\hline \multirow[t]{4}{*}{ Background } & $\begin{array}{l}\text { Studies of inpatient and outpatient teams suggest that relationships among providers have an important effect on } \\
\text { patient outcomes. }\end{array}$ \\
\hline & $\begin{array}{l}\text { Relationships among providers influence the way they communicate. This in turn influences the way they make sense } \\
\text { of what is happening with their patients (sensemaking) and react in uncertain clinical situations (improvising). }\end{array}$ \\
\hline & $\begin{array}{l}\text { Physician team Relationships, Improvising, and Sensemaking have been associated with outcomes for hospitalized patients, } \\
\text { including length of stay and complication rates. }\end{array}$ \\
\hline & $\begin{array}{l}\text { PRISm is a structured communication tool based on observations of effective inpatient teams. Its purpose is to improve patient } \\
\text { outcomes by changing the ways that physicians communicate and improving their ability to make sense and improvise. }\end{array}$ \\
\hline \multirow{5}{*}{ Intervention } & The PRISm communication tool has three components: \\
\hline & 1. Briefings before rounds - to determine how to round most efficiently \\
\hline & $\begin{array}{l}\text { 2. Structured patient discussions-using the "Situation, Task, Intent, Concern, } \\
\text { Calibrate (STICC)" framework }\end{array}$ \\
\hline & $\begin{array}{l}\text { 3. Debriefings after rounds - to organize care around what needs to be done, } \\
\text { who will do it, and ensure high priority tasks are completed }\end{array}$ \\
\hline & $\begin{array}{l}\text { PRISm is not a replacement for rounds. Instead, it should be integrated with rounds } \\
\text { to help you round } \\
\text { more effectively, improve patient discussions, and get all the work done. }\end{array}$ \\
\hline \multirow{21}{*}{$\begin{array}{l}\text { Implementing } \\
\text { PRISm }\end{array}$} & Before daily rounds, ask one or more of the following questions: \\
\hline & -Who is our sickest patient today? \\
\hline & - Did anyone have a change overnight? \\
\hline & -Who do we need to see first? \\
\hline & - Do we have any early admits/discharges? \\
\hline & - Do we have everything we need for rounds? \\
\hline & This quick, 5-min discussion will help to guide more efficient rounds. \\
\hline & While discussing individual patients, think about: \\
\hline & Situation: What are we dealing with? \\
\hline & Task: What do we need to do? \\
\hline & Intent: Why are we doing it? \\
\hline & Concern: What are we watching for? \\
\hline & Calibrate: What don't we know? What do we do if...? \\
\hline & STICC can be used during each patient discussion, or only for specific patients. \\
\hline & Ask these questions when you think they would improve patient discussions. \\
\hline & End daily rounds by asking: \\
\hline & -What are our biggest priorities? \\
\hline & - Who do we need to talk to? \\
\hline & -Who is going to do what? \\
\hline & • How can we help each other get things done? What can I do? \\
\hline & This 5-min recap ensures all tasks are done most efficiently. \\
\hline
\end{tabular}

briefings and debriefings to a combined 10 to $15 \mathrm{~min}$. We stress that the briefing should help the team to conduct rounds more effectively, and the debriefing should help save time later in the day.

The pocket guide (Figure 1) is a resource that attendings can use to easily prompt the PRISm structured communications. It is intended as a tool to facilitate the structured communication not to prompt people to deliver certain care for certain patients. It outlines exactly what is expected in the briefings and debriefings and includes the STICC components to guide individual patient discussions. Because our goal is for PRISm to be selfreinforcing through its positive impact on workflow and patient care, we expect it to become integrated into rounds in a way that will make use of the pocket guide unnecessary on an ongoing basis. Our goal is to create sufficient structure for briefings, debriefings, and STICC to achieve intervention consistency while also allowing each team sufficient flexibility to use PRISm most effectively

We are sending the information sheet and pocket guide to each participating attending, then meeting with them for approximately $20 \mathrm{~min}$ to review the PRISm 


\section{PRISm pocket guide Improve Physician team Relationships, Improvising, Sensemaking}

\section{Briefings}

start rounds by asking:

- Who is our sickest patient today?

- Did anyone have a change overnight?

- Who do we need to see first?

- Do we have any early admits / discharges?

- Do we have everything we need for rounds?

\section{Debriefings}

End rounds by asking:

- What are our biggest priorities?

- Who do we need to talk to?

- Who is going to do what?

- How can we help each other get things done? What can I do?

\section{PRISm pocket guide}

Improve Physician team Relationships, Improvising, Sensemaking

\section{STICC}

\section{For patient discussions}

Situation: what are we dealing with?

Task: what do we need to do?

Intent: $\quad$ why are we doing it?

Concern: what are we watching for?

Calibrate: what don't we know?

what do we do if...?

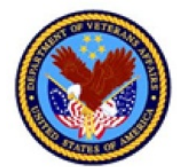

Figure 1 PRISm pocket guide.

information sheet and pocket guide and answer questions regarding implementation. We will send participants background literature in advance if they are interested in reading it $[15,19,31,32]$, but their participation and use of the tool will not be contingent on this having been done.

\section{Assessment of PRISm uptake}

We are observing rounds to assess PRISm uptake, making field notes and audiotaping rounds for further review. Each day, we assess whether briefings and debriefings occurred and what questions were used most frequently. We also note whether the STICC framework was incorporated into discussions of individual patients and for which types of patients STICC was most likely to be used.

For each team, we will assess the proportion of time that briefings, STICC elements, and debriefing were used and then aggregate these metrics across all teams for an overall assessment of its feasibility and utility. The field note template containing the uptake data collection is in Additional file 1. The template also contains the elements that will be used to assess team outcomes, described below in our assessment of outcomes

\section{Assessment of team outcomes}

We will assess physician team outcomes to allow us to assess the impact of PRISm on the teams and on their patients' outcomes. While we do not expect to have power to assess statistical significance, trends will be helpful in developing sample size estimates for a larger implementation trial. Additionally, we can assess the feasibility of our outcome data collection strategies for a multi-site trial.

We will assess the PRISm impact on provider relationships, improvising, and sensemaking in two ways. First, we will administer the work relationship survey (Additional file 2), a 15-item Likert survey developed by members of our research team in VA settings [33]. We will administer the survey to all physician team members (attending and residents) at the end of weeks 1 and 2, examining week 2 absolute scores as well as the degree of change in scores before and after PRISm, adjusting for initial scores.

Second, we will observe rounds daily using the field note guide as already described (Additional file 1). This guide contains the following elements:

- Relationships behaviors: We will assess relationship behaviors reflecting the seven characteristics in the Lanham framework to assess relationships [22].

- Improvising behaviors: We will assess improvising through assessing variability in daily activity, including order of rounds and time spent with each patient. We will also note whether we see 
improvising during our observations of discussions of individual patients for whom teams are not certain of the diagnosis.

- Sensemaking behaviors: We will use observation to assess sensemaking with regard to the way the team makes sense of their overall activities. We will examine how rounds were conducted, the order in which patients are discussed, and instances of communication with providers outside of the team.

We will assess sensemaking in discussions of individual patients using audiotaped discussions of individual patients. We will identify two to three complex patients most at risk for complications or poor outcomes based on CharlsonDeyo comorbidity scores and presenting complaints [34]. We will review audiotaped discussions of those patients on rounds, assessing team sensemaking using STICC elements. This analysis will be distinct from the assessment of STICC uptake. To assess uptake, we will assess the use of STICC across all patients as a measure of each team's uptake of PRISm; to assess the impact of STICC on sensemaking, we will examine each team's discussions of a similar group of the most complex patients as a measure of sensemaking. Because teams care for approximately 10 to 20 patients/week, identifying this number of complex patients is feasible.

We will analyze our observations to identify the ways that rounds are conducted before and after PRISm implementation, looking for changes in behaviors that reflect relationships, improvising, and sensemaking. We will assess each of these outcomes for the week prior to PRISm implementation and the week during implementation, comparing the results for each of these weeks. We anticipate comparing relationship survey score, number and types of behaviors reflecting relationship characteristics, order of rounds and time spent on patient discussions, and STICC components used during each week.

\section{Assessment of patient outcomes}

We will assess the PRISm impact on patient outcomes using the Institute for Healthcare Improvement (IHI) trigger tool [35]. The IHI trigger tool is a chart review tool developed to standardize assessment and measurement of adverse events in hospitals and objectively categorize their impact. It has been used in other studies to assess rates of harm. [2] The trigger tool contains several different modules that can be applied to chart review in a range of clinical settings. We propose using three modules that are most related to inpatient medical and surgical care: the care module, medication module, and surgical module. Reviews will be conducted as recommended by the IHI. Potential adverse events will be reviewed by the physician on the research team who did not conduct the initial review in a blinded fashion, without the reviewer knowing from what time period the potential event occurred by printing information and removing identifiers. We will categorize event severity using IHI categories E through I, ranging from "capacity for harm" to death.

While we do not anticipate having an adequate sample size to definitively compare outcomes of patients before and during PRISm implementation, we hope to begin to get a sense of the types of differences we may observe. We will analyze the association between our measures of relationships, improvising, and sensemaking, and the number and types of IHI trigger tool events before and after implementation across teams, adjusting for team workload and patient comorbidity and accounting for clustering using $t$-tests and logistic regression.

\section{Attending physician feedback}

At the beginning of the week following the PRISm implementation, we will interview the attending to obtain feedback on PRISm, starting with orientation, information sheet, and pocket guide and continuing through the implementation process. Interview questions are listed in Table 3.

We chose the week after implementation to allow the attending time to reflect on PRISm, while being able to provide feedback from the vantage point of having just implemented it. We may also ask other targeted questions based on what we observe. We anticipate that the entire interview will last 30 to $45 \mathrm{~min}$. We are audiotaping responses and taking notes during the interview to capture key elements of the responses. Immediately after each interview, we create a summary of the interview, using the audiotape to review any areas that were not clear.

We will analyze interview responses and summaries to identify themes regarding improving PRISm. For orientation and initial implementation, we will examine what was most useful, what ought to be improved, or what ought to be included. For the briefings, STICC, and debriefings, we will also assess their impressions of how PRISm influences rounds and patient discussions, as this feedback may also help us refine PRISm. In addition to the interview, we also believe that our observations will be important for obtaining information to refine PRISm, such as what practices or questions worked particularly well or poorly. The observations will also provide context for the attending interview responses. We will triangulate the interview responses with our observational data, comparing attending feedback with their use patterns.

\section{Trial status}

A trial of the PRISm intervention has been funded by the Veterans Affairs Health Services Research and Development Quality Enhancement Research Initiative. PRISm has been approved by the Internal Review Board of the University of Texas Health Science Center at San 
Antonio and the Research and Development committee at the South Texas Veterans Health Care System. We have implemented PRISm on two inpatient medicine physician teams.

\section{Discussion}

Our study has the potential to help us understand how physician relationships, improvising, and sensemaking can be leveraged to improve the care of hospitalized patients. These insights will improve our understanding of the ways physicians navigate the complex, uncertain, and unpredictable clinical settings in which they work through how they relate, improvise, and make sense of what is happening as they take care of patients. Because of the high frequency and cost of adverse events among hospitalized patients, dissemination and implementation of the PRISm intervention could have a significant positive impact on hospital care and resource utilization.

This study could also contribute new understanding of how sensemaking and improvising intersect with clinical reasoning. Historically, clinical reasoning has been conceptualized as an individual level activity. However, emerging frameworks in the clinical reasoning literature, including distributed cognition and situativity theory, emphasize the social, shared nature of coming to an understanding of a patient's diagnosis and treatment plan [36]. In this regard, clinical reasoning has similarities to sensemaking. We view sensemaking, though, as larger in scope than clinical reasoning in that it encompasses other aspects of care, such as safety and psychosocial factors.

Improvising also involves considerable human infrastructure, including practices, expertise, and knowledge of the rules for collaborating that enable team members to influence the quality of their improvisational processes [19]. "When a team of improvisers pays close attention to each other, hearing and remembering everything, and respecting all that they hear, a group mind forms." [37] The goal then is to harness this group mind for the good of the patient. We believe this study could contribute new insights about how improvising in inpatient teams intersects with clinical reasoning, including ideas about where the boundaries around improvising in care teams and clinical reasoning may exist.

The PRISm intervention may enable teams to more effectively develop a shared mental model [38] of not only each individual patient's treatment plan but also the needs and priorities for their entire set of patients. This shared mental model may be an important aspect of preventing errors and recognizing complications early. The ability to create a shared mental model may extend beyond the team to other providers, patients, and caregivers, influencing the team's ability to come to better understandings of multidisciplinary aspects of care and post-discharge care planning. Finally, this shared understanding improves providers' ability to learn, or change their mental models, enabling them to improve their knowledge, skills, and patient care.

While our study is focused on a small number of teams, we will be collecting rich, in-depth data that will allow us to understand how relationships, improvising, and sensemaking influence care, and what approaches to influence these team attributes may be most effective in larger-scale studies. In addition to enhancing our understanding of relationships, improvising, and sensemaking; their association with patient outcomes; and their intersection with clinical reasoning, our results could form the basis for a larger follow-up implementation trial. 


\section{Additional files}

Additional file 1: Daily field note guide. The field note guide contains the template for daily observational data collection.

Additional file 2: Work relationship scale. The work relationship survey is a 15-item Likert survey.

\section{Competing interests}

The authors declare that they have no competing interests.

\section{Authors' contributions}

All authors were involved in the design of this intervention. LL wrote the initial draft of this manuscript, and all authors provided substantive comments and edits, producing the final version. All authors read and approved the final manuscript.

\section{Acknowledgements}

We would like to thank the physicians who are participating in this study.

\section{Author details}

${ }^{1}$ South Texas Veterans Health Care System, Texas, USA. ${ }^{2}$ School of Medicine, University of Texas Health Science Center at San Antonio, 7703 Floyd Curl Drive, 373 L, San Antonio, Texas 78229, USA. ${ }^{3}$ The University of Texas at Austin, Austin, Texas, USA.

Received: 17 October 2014 Accepted: 6 November 2014

Published online: 26 November 2014

\section{References}

1. The Institute of Medicine: To Err is Human: Building a Safer Health System. Washington DC: The National Academies Press; 2000

2. Landrigan CP, Parry GJ, Bones CB, Hackbarth AD, Goldmann DA, Sharek PJ: Temporal trends in rates of patient harm resulting from medical care. NEJM 2010, 323(22):2124-2135.

3. Krauss MJ, Nguyen SL, Dunagan WC, Birge S, Constantinou E, Johnson S, Caleca B, Fraser VJ: Circumstances of patient falls and injuries in 9 hospitals in a mid-western healthcare system. Infection Control Hosp Epi 2007, 28(5):544-550.

4. Hurd T, Posnett J: Point prevalence of wounds in a sample of acute hospitals in Canada. Int Wound J 2009, 6(4):287-293.

5. Garcin F, Leone M, Antonini F, Charvet A, Albanese J, Martin C: Non-adherence to guidelines: an avoidable cause of failure of empirical antimicrobial therapy in the presence of difficult-to-treat bacteria. Intensive Care Med 2010, 36(1):75-82.

6. Williams SC, Schmaltz SP, Morton DJ, Koss RG, Loeb JM: Quality of care in US hospitals as reflected by standardized measures, 2002-2004. NEJM 2005, 353:255-264

7. Eber MR, Laxminarayan R, Perencevich EN, Malani A: Clinical and economic outcomes attributable to healthcare-associated sepsis and pneumonia. Arch Int Med 2010, 170(4):147-153.

8. Plsek P: Redesigning health care with insights from the science of complex adaptive systems. In Crossing the Quality Chasm: A New Heath System for the 21st Century. Washington DC: National Academy of Sciences; 2000:309-322.

9. Bar Yam Y: Making Things Work: Solving Complex Problems in a Complex World. United States of America: NECSI Knowledge press; 2004.

10. Cilliers P: Complexity and Postmodernism: Understanding Complex Systems. New York, NY: Routledge; 1998.

11. Weick KE, Sutcliffe KM: Managing the Unexpected: Assuring High Performance in an Age of Complexity. Ann Arbor, Michigan: University of Michigan Business School Series; 2001.

12. Plsek PE, Greenhalgh T: Complexity science: the challenge of complexity in health care. BMJ 2001, 323(7313):625-628.

13. Han PK, Klein WM, Arora NK: Varieties of uncertainty in health care: a conceptual taxonomy. Med Decision Making 2011, 31(6):282-38.

14. Leykum LK, Pugh J, Lawrence V, Parchman M, Noel PH, McDaniel RR: The importance of organizational and disease characteristics in improving outcomes of patients with chronic disease: a systematic review of congestive heart failure and comparison with diabetes. Imp Sci 2010, 25(5):66.

15. Weick KE: Sensemaking in Organizations. Thousand Oaks, CA: Sage Publications; 1995.
16. Blatt $R$, Christianson MK, Sutcliffe KM, Rosenthal MM: A sensemaking lens on reliability. J Organiz Behav 2006, 27:897-917.

17. Ghaferi AA, Birkmeyer JD, Dimick JB: Complications, failure to rescue, and mortality with major inpatient surgery in medicare patients. Ann Surg 2009, 250(6):1029-1034.

18. Haidet P: Jazz and the 'art' of medicine: improvisation in the medical encounter. Annals Fam Med 2007, 5(3):164-169.

19. McKenna K, Leykum LK, McDaniel RR: The role of improvising in patient care. Health Care Manag Rev 2013, 38(1):1-8.

20. Miller WL, McDaniel RR Jr, Crabtree BF, Stange KC: Practice jazz: understanding variation in family practices using complexity science. J Fam Pract 2001, 50(10):872-878.

21. Edmondson A: Speaking up in the operating room: how team leaders promote learning in interdisciplinary action teams. J Manag Stud 2003, 40(6):1419-1452.

22. Lanham HJ, McDaniel RR, Crabtree BF, Miller WL, Stange KC, Tallia A, Nutting PA: How improving practice relationships among clinicians and nonclinicians can improve quality in primary care. Jt Comm J Qual Patient Saf 2009, 35(9):457-466.

23. Stroebel CK, McDaniel RR Jr, Crabtree BF, Miller WL, Nutting PA, Stange KC: How complexity science can inform a reflective process for improvement in primary care practices. Jt Comm J Qual Patient Saf 2005, 31(8):438-446.

24. Jordan ME, Lanham HJ, Crabtree BF, Nutting PA, Miller WL, Stange KC, McDaniel RR: The role of conversation in health care interventions: enabling sensemaking and learning. Implement Sci 2009, 4:15.

25. Manthous CA, Hollingshead AB: Team science and critical care. Am J Respir Crit Care Med 2011, 184:17-25.

26. McAllister C, Leykum LK, Lanham HJ, Reisinger $\mathrm{H}$, Kohn JL, Palmer RF, Pezzia C, Agar M, Parchman ML, Pugh JA, McDaniel RR: The impact of inpatient physician team relationships on outcomes of hospitalized patients. J Hosp Med, in press.

27. Leykum LK, Kumar P, Parchman ML, McDaniel RR, Lanham HJ, Agar M: Use of agent-based model to understand clinical systems. J Artif Societies Social Simul 2012, 15(3):2.

28. Anderson RA, Ammarell N, Bailey D Jr, Colon-Emeric C, Corazzini KN, Scotten LM, Piven ML, Utley-Smith Q, McDaniel RR: Nurse assistant mental models, sensemaking, care actions, and consequences for nursing home residents. Qual Health Res 2005, 15(8):1006-1021.

29. Scott JG, Cohen D, Dicicco-Bloom B, Miller WL, Stange KC, Crabtree BF: Understanding healing relationships in primary care. Ann Fam Med 2008, 6(4):315-322.

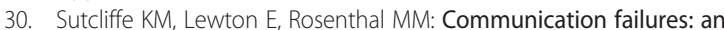
insidious contributor to medical mishaps. Acad Med 2004, 79:186-194.

31. Neily J, Mills PD, Young-Xu Y, Carney BT, West P, Berger DH, Mazzia LM, Paull $D E$, Bagian JP: Association between implementation of a medical team training program and surgical mortality. JAMA 2010, 304(15):1693-1700.

32. Dixon-Woods M, Bosk CL, Aveling EL, Goeschel CA, Pronovost PJ: Explaining Michigan: developing an ex post theory of a quality improvement program. Milbank Q 2011, 89(2):167-205.

33. Finley EPPJ, Lanham HJ, Leykum L, Veerapaneni P, Parchman ML: Relationship quality and patient-assessed quality of care in VA primary care clinics: development and validation of the Work Relationships Scale. Ann Fam Med 2013, 11(6):543-549.

34. Deyo RA, Cherkin DC, Ciol MA: Adapting a clinical comorbidity index for use with ICD-9-CM administrative databases. J Clin Epidemiol 1992, 45(6):613-619.

35. Griffin FA, Resar RK: IHI Global Trigger Tool for Measuring Adverse Events (Second Edition), IHI Innovation Series white paper. Cambridge, MA: Institute for Healthcare Improvement; 2009. Available on www.IHI.org

36. Durning SJ, Artino AR: Situativity theory: a perspective on how participants and the environment can interact: AMEE guide no. 52. Med Teach 2011, 33:188-199.

37. Vera $D$, Crossan M: Improvisation and innovative performance in teams. Org Sci 2005, 16:203-224

38. Nonaka I: A dynamic theory of organizational knowledge creation. Org Sci 1994, 5(1):14-37.

\section{doi:10.1186/s13012-014-0171-3}

Cite this article as: Leykum et al:: Improving outcomes of hospitalized patients: the Physician Relationships, Improvising, and Sensemaking intervention protocol. Implementation Science 2014 9:171. 